\title{
An Incidental Finding of Hepatoblastoma Due to Bleeding After a Fall From Rollerblades
}

Alessandra Guiner, MD ${ }^{1,2}$ - Janelle McCall, MD ${ }^{1,2}$

A 10-year-old boy with no significant medical history presented for evaluation after an unwitnessed fall forward from rollerblades onto asphalt. The patient reported no loss of consciousness after the fall and was able to ambulate home. Within an hour, he had developed 3 episodes of nonbloody, nonbilious emesis, vague abdominal pain, and became sleepier, according to his mother. He was brought in by emergency medical services to a level 1 pediatric trauma center, with a cervical collar and backboard in place. He was given a fluid bolus and ondansetron en route to the center.

\section{Physical examination}

On arrival (about an hour after the fall), the patient was tachycardic (heart rate, 148 bpm) and tachypneic (respiratory rate, 24 breaths/min), with a normal blood pressure and oxygen saturation level.

Findings from the physical examination revealed that the boy was well nourished and alert (Glasgow Coma Scale score, 15), but he was exhibiting excessive sleepiness. He also had generalized abdominal pain with minimal guarding but no distension or overlying bruising. The remainder of the physical examination was unremarkable.

\section{Diagnostic testing \\ Bedside testing showed a point-of-care} hemoglobin level of $10.1 \mathrm{~g} / \mathrm{dL}$ and a positive Focused Assessment with Sonography for Trauma (FAST) test, with concern for fluid seen in Morison pouch/hepatorenal recess. Other laboratory test results showed a normal complete blood cell count, normal electrolyte level, normal liver enzyme level, and normal lipase levels. Because the patient was hemodynamically stable, a computed tomography (CT) scan of the abdomen and pelvis was obtained (Figures $1 \mathrm{a}$ and $1 \mathrm{~b}$ ), showing a large-volume hemoperitoneum with a round, actively-bleeding mass measuring $5 \times 5.3 \mathrm{~cm}$ in the left hepatic lobe. The CT scan was interpreted by the radiologist as developing hematoma vs a primary hepatic mass with subsequent hemorrhage.

\section{Treatment and management}

The patient was stabilized with fluids and 2 units of packed red blood cells ( $p R B C s$ ) prior to embolization of the hepatic artery by an interventional radiologist, after which the patient required multiple $\mathrm{pRBC}$ transfusions, fresh frozen plasma, and platelets because of continued bleeding in the first 24 hours

\section{AFFILIATIONS:}

'Department of Pediatric Emergency Medicine, UT Southwestern Medical Center, Dallas, Texas

${ }^{2}$ Department of Pediatric Emergency Medicine, Children's Health, Dallas, Texas

\section{CITATION:}

Guiner A, McCall J. Hepatoblastoma caused by bleeding after a fall from rollerblades. Consultant. Published online May 24, 2021. doi:10.25270/con.2021.05.00007

Received January 1, 2021. Accepted April 1, 2021.

\section{DISCLOSURES:}

The authors report no relevant financial relationships.

\section{CORRESPONDENCE:}

Janelle McCall, MD, Children's Health, 1935 Medical District Drive, Dallas, TX 75235

(Janelle.McCall@UTSouthwestern.edu)

post-surgery. During the patient's hospital stay, he underwent a biopsy of the hepatic mass, which revealed a hepatoblastoma. The patient was determined to be high risk because of his age and elevated initial alpha-fetal protein (AFP) level. He received 6 cycles of chemotherapy and underwent a lobectomy (left hepatectomy and cholecystectomy) with negative tumor margins after 3 months. He later received consolidation chemotherapy. Recent scans showed no evidence of disease at a 1-year follow-up visit.

\section{Discussion}

In pediatric care, blunt trauma is more prevalent than penetrating trauma, with penetrating injuries accounting for only $10 \%$ to $20 \%$ of all trauma admissions at trauma centers.' Blunt trauma commonly results from motor vehicle accidents or falls from large heights; however, this case highlights a patient with a minor traumatic mechanism presenting with significant pain and somnolence. The differential diagnosis included intracranial bleed, solid organ injury, and intestinal injury. He underwent a full trauma workup, including laboratory testing because of physical findings of tachycardia, excessive sleepiness, and abdominal pain, which did not match his history. Of note, the positive FAST examination expedited the CT scan, which resulted in the diagnosis of an incidental tumor.

Although the FAST examination is found to be less accurate in children than in adults, it is still shown to be useful, especially in conjunction with physical examination and laboratory findings to expedite CT scanning. A meta-analysis of studies examining the use of FAST examinations in pediatric care revealed a sensitivity of $66 \%$ (95\% Cl, 56\%-75\%), specificity of $95 \%$ (95\% Cl, 93\%-97\%), and negative likelihood ratio of $0.36(95 \% \mathrm{Cl}, 0.27-0.47)$. 

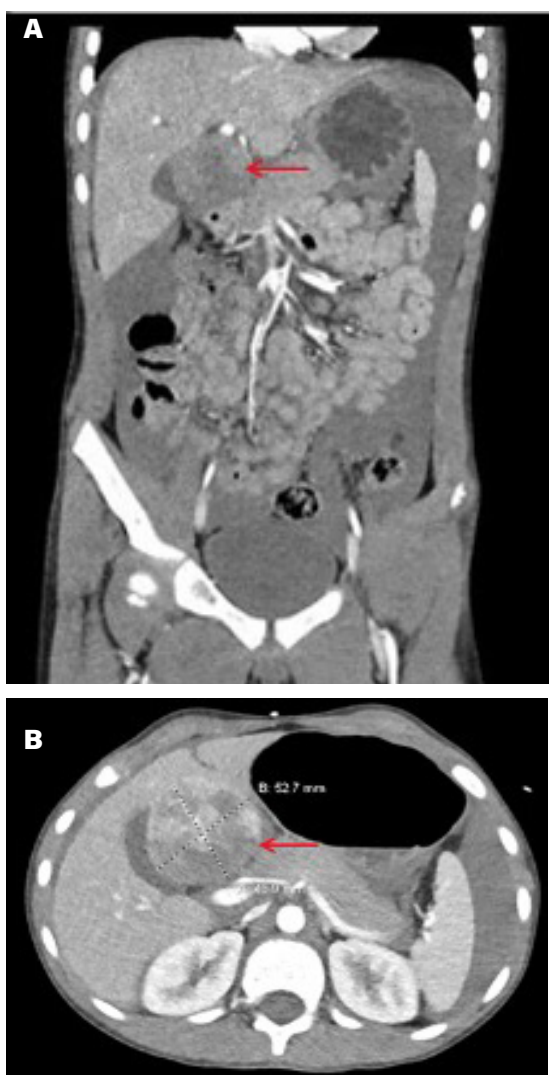

Figure 1. Coronal (a) and axial (b) views of the CT abdomen/pelvis showing a large volume hemoperitoneum with a round, actively-bleeding mass measuring $5 \times 5.3 \mathrm{~cm}$ in segment IVb of the left hepatic lobe (red arrows).

There are very few cases in the pediatric care literature about symptomatic new tumor findings caused by trauma. There have been some cases published in the recent past that involved diagnosis of Wilms tumor caused by a tumor bleed or kidney rupture in children with minor trauma and several similar reports in the adult care literature. ${ }^{3-5}$

Often, CT scans obtained to screen for trauma show incidental findings, which may be clinically significant. However, these tumors rarely cause symptoms in the acute setting of concurrent trauma. In a 2020 review article of incidental brain tumors in the pediatric population that included 14 studies representing more than 300 patients, the authors found that $23 \%$ of the tumors were diagnosed via head imaging obtained because of trauma. ${ }^{6}$
Our patient was diagnosed with hepatoblastoma, which is the most common malignant liver tumor in children, but it is still exceedingly rare, comprising approximately $1 \%$ of all childhood cancers. ${ }^{78}$ It is most frequently diagnosed in patients younger than 5 years of age, while older pediatric patients (5-18 years) comprise only $5 \%$ to $7 \%$ of cases. ${ }^{9,10}$ Hepatoblastoma is typically idiopathic, but it can be associated with certain conditions such as Beckwith-Wiedemann, Aicardi syndrome, familial adenomatous polyposis, Goldenhar syndrome, Li-Fraumeni syndrome, trisomy 18 , trisomy 21 , and type 1a glycogen storage disease (von Gierke disease). ${ }^{8}$

Treatment of hepatoblastoma typically includes chemotherapy and complete surgical resection, and unresectable hepatoblastoma can be treated with liver transplantation." Poor prognosis is predicted by tumor size greater than $5 \mathrm{~cm}$, metastatic disease at time of diagnosis, elevated AFP level, older patient age, lack of surgical resection, and African American ethnicity. ${ }^{9,10}$ Over the past several decades, there have been significant advances in the treatment of hepatoblastoma, and currently the 5 -year survival rates are $80 \%$, a significant improvement from the $60 \%$ previously reported prior to 2009. ${ }^{9,10}$

\section{Conclusion}

Incidental findings of clinically significant or symptomatic tumors are rare but may present because of a traumatic event that incites bleeding of an already fragile and over-vascularized site. Pain out of proportion to the history should be an indication for further workup in a patient with trauma. A FAST examination may be used as a screening tool to evaluate for free fluid in the abdomen after a trauma; however, CT scanning is indicated for definitive diagnosis. In our case, the FAST examination expedited the CT scan, which identified a hepatoblastoma, a rare tumor with high survival rates mainly affecting young children.

\section{REFERENCES}

1. Cotton BA, Nance ML. Penetrating trauma in children. Semin Pediatr Surg. 2004;13(2):8797. https://doi.org/10.1053/j.sempedsurg.2004.01.004

2. Holmes JF, Gladman A, Chang $\mathrm{CH}$. Performance of abdominal ultrasonography in paediatric blunt trauma patients: a meta-analysis. J Pediatr Surg. 2007;42:1588-1594. https://doi. org/10.1016/j.jpedsurg.2007.04.023

3. Dunlop JH, Cohen JS. Incidental renal mass found on focused assessment with sonography in trauma. Pediatr Emerg Care. 2014;30(10):752-754. https://doi.org/10.1097/ PEC.0000000000000242

4. Gallagher R, Vieria R, Levy J. Bedside ultrasonography in the pediatric emergency department the focused assessment with sonography in trauma examination uncovers an occult intraabdominal tumor. Pediatr Emer Care. 2012;28(10):1107-1111. https://doi. org/10.1097/PEC.0b013e31826d1e86

5. Gökçe AH. Gastrointestinal stromal tumor leading to acute abdomen and hypovolemic shock in a trauma patient. Ulus Travma Acil Cerrahi Derg. 2019;25(1):83-85. https://doi. org/10.5505/tjtes.2018.35005

6. Jumah F, Rallo MS, Quinoa T, et al. Incidental Brain tumors in the pediatric population: a systematic review and reappraisal of literature. World Neurosurg. 2020;139:121-131. https://doi. org/10.1016/j.wneu.2020.02.178

7. Finegold MJ, Egler RA, Goss JA, et al. Liver tumors: pediatric population. Liver Transpl. 2008;14(11):1545-1556. https://doi. org/10.1002/lt.21654

8. Litten JB, Tomlinson GE. Liver tumors in children. Oncologist. 2008;13(7):812-820. https:// doi.org/10.1634/theoncologist.2008-0011

9. Allan BJ, Parikh PP, Diaz S, Perez EA, Neville $\mathrm{HL}$, Sola JE. Predictors of survival and incidence of hepatoblastoma in the paediatric population. HPB (Oxford). 2013;15(10):741-746. https://doi.org/10.1111/hpb.12112

10. Feng J, Polychronidis G, Heger U, Frongia G, Mehrabi A, Hoffman K. Incidence trends and survival prediction of hepatoblastoma in children: a population-based study. Cancer Commun. 2019;39(1):62. https://doi.org/10.1186/ s40880-019-0411-7

11. Kremer N, Walther AE, Tiao GM. Management of hepatoblastoma: an update. Curr Opin Pediatr. 2014;26(3):362-369. https://doi. org/10.1097/MOP.0000000000000081 